\title{
Tradeoff between energy and error in the discrimination of quantum-optical devices
}

\author{
Alessandro Bisio, ${ }^{1,2}$ Michele Dall'Arno, ${ }^{1,2}$ and Giacomo Mauro D'Ariano ${ }^{1,2}$ \\ ${ }^{1}$ Quit group, Dipartimento di Fisica "A. Volta", via Bassi 6, 27100 Pavia, Italy \\ ${ }^{2}$ Istituto Nazionale di Fisica Nucleare, Gruppo IV, via Bassi 6, 27100 Pavia, Italy
}

(Dated: November 21, 2018)

\begin{abstract}
We address the problem of energy-error tradeoff in the discrimination between two linear passive quantum optical devices with a single use. We provide an analytical derivation of the optimal strategy for beamsplitters and an iterative algorithm converging to the optimum in the general case. We then compare the optimal strategy with a simpler strategy using coherent input states and homodyne detection. It turns out that the former requires much less energy in order to achieve the same performances.

PACS numbers:
\end{abstract}

\section{INTRODUCTION}

There are many contexts in which one wishes to use as little energy as possible in order to perform a desired task. Consider the case in which one wants to gather information about an unknown device. The most general approach to the problem is to probe the target and to measure its response. This implies an undesired perturbation of the system. Often such perturbation depends on the energy of the probe, and one can ask what is the minimum amount of energy the probe must have in order to perform the desired task. Exploiting the distinctive features of quantum theory, such as entanglement, one could in principle achieve better performances with respect to a classical strategy with the same energy. For example, in the context of quantum illumination [1 3 ], one discovers that the use of entangled light allows to enhance the detection and the imaging of an object.

The task we are considering in this paper is the discrimination between two quantum optical devices (such as beamsplitters or phase shifters). An optical device is represented by a unitary transformation $U$ acting on a system described by a Fock space $\mathcal{H}$. The general problem in which one wants to discriminate between two unitaries has been widely investigated in the literature [4-8] and can be summarized as follows. Suppose we are provided with a single use [9] of an unknown device randomly chosen in a set of two $U_{1}$ and $U_{2}$, with equal prior probability. Our task is to distinguish between $U_{1}$ and $U_{2}$ with a probability of error not greater than a given threshold $q$. One sends an input state $|\psi\rangle$ that undergoes the unitary evolution described either by $U_{1}$ or $U_{2}$. Finally, one applies the optimal POVM [10] in order to distinguish the two possible output states $U_{1}|\psi\rangle$ or $U_{2}|\psi\rangle$ with minimum error probability. This error probability depends on the input $|\psi\rangle$, so one can optimize on $|\psi\rangle$. In the case at hand, $U_{1}$ and $U_{2}$ describe optical devices and the input state $|\psi\rangle$ is a quantum state of light, with a well-defined mean value of the energy. Since in general many states allow to discriminate with an error probability $P_{e} \leq q$, one can wonder which state $\left|\psi^{*}\right\rangle$ accomplishes this task with minimum mean value of the energy.
Here we analyze the energy-error tradeoff in the case in which the optical devices to be discriminated are linear, passive and lossless. This discrimination is useful for example when reading classical digital information encoded in the reflectivity of a media, such as conventional CDs or DVDs. This particular application has first been suggested in [11] for the discrimination of quantum channels affected by loss and noise. In particular, there it is shown that, for fixed mean number of photons irradiated, non-classical light can outperform any classical source in terms of the amount of information retrieved. Recently, the discrimination of lossy beamsplitters has been considered in [12], in the scenario where one has access to only one of the input and output modes and to a restricted class of input states. A similar scenario, namely the discrimination of a lossy quantum channel from an ideal one, has been studied also in [13].

The paper is organized as follows. In Section [I] we review some basic notions of linear optics and introduce the problem. The analytical derivation of the energyerror tradeoff when the devices are beamsplitters is given in Section III A, while a numerical algorithm for the solution in the general case is presented in Section IIIB. In Section III C we analyze the energy-error tradeoff in the restricted scenario in which only coherent states and homodyne detections are available. We will then quantify the advantages that one has by adopting the optimal quantum strategy. Section IV concludes the paper with a discussion of the results.

\section{DISCRIMINATION OF LINEAR PASSIVE OPTICAL DEVICES}

A $M$-modes quantum optical device [14, 15] is described by a unitary operator $U$ relating $M$ input optical modes with annihilation operators $a_{i}$ on $\mathcal{H}_{i}$, to $M$ output optical modes with annihilation operators $a_{i}^{\prime}$ on $\mathcal{H}_{i^{\prime}}$, where $\mathcal{H}_{i}$ denotes the Fock space of the optical mode $i$. We denote as $\mathcal{H}=\bigotimes_{i} \mathcal{H}_{i}$.

An optical device is called linear if the operators of the output modes are related to the operator of the input 
modes by a linear transformation, namely

$$
a^{\prime}:=U a_{i} U^{\dagger}=\sum_{j=1}^{N} A_{i j} a_{j}+\sum_{j=1}^{N} B_{i j} a_{j}^{\dagger}, \quad i=1, \ldots, N .
$$

The above equation can be rewritten in the more compact form

$$
\left(\begin{array}{c}
\mathbf{a}^{\prime} \\
\mathbf{a}^{\prime \dagger}
\end{array}\right)=S\left(\begin{array}{c}
\mathbf{a} \\
\mathbf{a}^{\dagger}
\end{array}\right)
$$

where $S$ is the $2 N \times 2 N$ scattering matrix defined as

$$
S:=\left(\begin{array}{cc}
A & B \\
\bar{B} & \bar{A}
\end{array}\right)
$$

( $\bar{X}$ is the complex conjugate of $X), \mathbf{a}=\left(a_{1}, \ldots a_{N}\right)$ is the vector of annihilation operators of the input mode, and analogously $\mathbf{a}^{\prime}$ for the output modes. If $B=0$ in Eq. (2) the device is called passive and conserves the total number of photons, that is

$$
\langle\psi|N| \psi\rangle=\left\langle\psi\left|U^{\dagger} N U\right| \psi\right\rangle
$$

with $N:=\sum_{i} a_{i}^{\dagger} a_{i}$ the number operator on $\mathcal{H}$.

Suppose now that we want to discriminate between two linear optical passive devices $U_{1}$ and $U_{2}$. If a single use of the unknown device is available, the most general strategy consists of i) preparing a bipartite input state $\rho \in \mathcal{B}(\mathcal{H} \otimes \mathcal{K})(\mathcal{K}$ is an ancillary Fock space with mode operators $\left.b_{i}\right)$, ii) applying locally the unknown device and iii) performing a bipartite POVM $\Pi=\left\{\Pi_{1}, \Pi_{2}\right\}$ on the output state $\left(\mathcal{U}_{x} \otimes \mathcal{I}_{\mathcal{K}}\right) \rho=\left(U_{x} \otimes I_{\mathcal{K}}\right) \rho\left(U_{x}^{\dagger} \otimes I_{\mathcal{K}}\right)(x$ can be either 1 or 2$)$

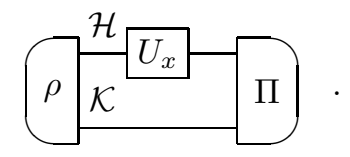

When the device is randomly chosen from the set $\left\{U_{1}, U_{2}\right\}$ with equal probabilities $p_{1}=p_{2}=1 / 2$, the minimum probability of error in the discrimination can be proved to be [16]

$$
P_{e}\left(\rho, U_{1}, U_{2}\right)=\frac{1}{2}\left(1-\left\|\left(\mathcal{U}_{1} \otimes \mathcal{I}_{\mathcal{K}}\right) \rho-\left(\mathcal{U}_{2} \otimes \mathcal{I}_{\mathcal{K}}\right) \rho\right\|_{1}\right) .
$$

where $\|X\|_{1}=\operatorname{Tr}\left[\sqrt{X^{\dagger} X}\right]$. If we define as $N_{\mathcal{K}}=\sum_{i} b_{i}^{\dagger} b_{i}$ the number operator on the ancillary modes $b_{i}$, the energy of the state $\rho$ is proportional to

$$
E(\rho):=\frac{1}{2}+\operatorname{Tr}\left[\rho\left(N \otimes I_{\mathcal{K}}+I \otimes N_{\mathcal{K}}\right)\right]
$$

while clearly the energy that flows through the unknown device is $E_{d}(\rho):=\frac{1}{2}+\operatorname{Tr}\left[\rho\left(N \otimes I_{\mathcal{K}}\right)\right]$.

Since we have $P_{e}\left(\left(\mathcal{U}_{1}^{\dagger} \otimes \mathcal{I}_{\mathcal{K}}\right) \rho, I, U_{2} U_{1}^{\dagger}\right)=P_{e}\left(\rho, U_{1}, U_{2}\right)$ and Eq. (3) implies $E\left(\left(\mathcal{U}_{1}^{\dagger} \otimes \mathcal{I}_{\mathcal{K}}\right) \rho\right)=E(\rho)$, we can restrict our analysis to the case in which $U_{1}=I$ and
$U_{2}=U$. We consider now the problem to find the minimum energy input state $\rho^{*}$ that allows us to discriminate between $I$ and $U$ with probability of error not greater than a given threshold $q$, that is

$$
\rho^{*}=\arg \min _{\rho \text { s.t. } P_{e}(\rho) \leq q} E(\rho)
$$

Sometimes one is more interested in minimizing the energy $E_{d}(\rho)$ flowing through the device, rather than the total energy $E(\rho)$ of the input state. In the following we show that no ancillary modes are required for optimal discrimination, so these minimization problems lead to the same optimal input state.

The following lemmas allow us to simplify Eq. (7).

Lemma 1. Without loss of generality the minimization in Eq. (7) can be rewritten as

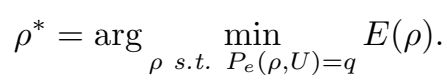

Proof. Suppose that for the optimal state $\rho^{*}$ one has $P_{e}(\rho)<q$. Since $P_{e}(\rho)$ is a continuous function there exists a $0<\alpha \leq 1$ such that $P_{e}((1-\alpha) \rho+\alpha|0\rangle\langle 0|)=q$, where $|0\rangle\langle 0|$ denotes the vacuum state. By observing that $E((1-\alpha) \rho+\alpha|0\rangle\langle 0|)<E(\rho)$ we have the thesis.

Lemma 2. The optimal state achieving the minimum in Eq. (7) can be chosen pure.

Proof. First, let us prove that the minimization of $E(\rho)$ for a given value of $P_{e}$ is equivalent to the minimization of the convex combination

$$
F(\rho)=p P_{e}(\rho)+(1-p) E(\rho)
$$

for a fixed value of $p$. Suppose that we find $\rho^{*}$ that minimizes $F(\rho)$. It is then clear that for $q:=P_{e}\left(\rho^{*}\right), E(\rho)$ gives the minimum possible value for the energy because any lower energy would decrease $F$. From Eq. (5) and the convexity of the trace distance, it follows that $P_{e}(\rho)$ is a concave function of $\rho$. Since $P_{e}(\rho)$ is concave and $E(\rho)$ is linear, $F(\rho)$ is a concave function of $\rho$ and its minimum is attained on the boundary of its dominion, $i$. e. for pure states.

Lemma 2 allows us to rewrite Eq. (5) as

$$
P_{e}=\frac{1}{2}\left(1-\sqrt{1-\left|\left\langle\psi\left|\left(U \otimes I_{\mathcal{K}}\right)\right| \psi\right\rangle\right|^{2}}\right) .
$$

We now prove that no ancillary modes are required.

Lemma 3. Without loss of generality, the minimum in (17) is achieved without using ancillary modes.

Proof. Due to Lemma 2 the input state can be written as $|\psi\rangle=\sum_{i} c_{i}|i\rangle\left|\chi_{i}\right\rangle$ where $|i\rangle$ is an orthonormal basis in $\mathcal{H}$ and $\left|\chi_{i}\right\rangle$ are normalized states in $\mathcal{K}$. If we define $\left|\psi^{\prime}\right\rangle:=\sum_{i} c_{i}|i\rangle|0\rangle(|0\rangle$ is the vacuum state $)$, it is easy to verify that $P_{e}(\psi)=P_{e}\left(\psi^{\prime}\right)$ while $E(\psi) \geq E\left(\psi^{\prime}\right)$. 
After these consideration and disregarding some irrelevant constant factors, Eq. (7) can be rewritten as

$$
\left|\psi^{*}\right\rangle=\arg \min _{|\psi\rangle \text { s.t. }|\langle\psi|U| \psi\rangle|=K}\langle\psi|N| \psi\rangle,
$$

where from Eq. (10) it follows that $K=\sqrt{4 q(1-q)}$.

\section{THE OPTIMAL TRADEOFF}

\section{A. The case of beamsplitters}

In this section we derive an analytical expression for the optimal energy-error tradeoff for the case in which the devices to be discriminated are two beamsplitters. A beamsplitter is a two-mode linear passive quantum optical device whose scattering matrix $S$ has the form

$$
S:=\left(\begin{array}{cc}
A & 0 \\
0 & \bar{A}
\end{array}\right) \quad A \in S U(2) .
$$

In the following we will use the basis $\{|n m\rangle\}$ with respect to which $U$ is diagonal i.e.

$$
A=\left(\begin{array}{cc}
e^{i \delta} & 0 \\
0 & e^{-i \delta}
\end{array}\right), \quad 0 \leq \delta \leq \pi
$$

With this choice, for any $|\psi\rangle=\sum_{n, m=0}^{\infty} \alpha_{n m}|n, m\rangle$, we have $U|n m\rangle=e^{i \delta(n-m)}|n m\rangle$ and it is easy to observe that $\langle\psi|U| \psi\rangle=\sum_{n, m=0}^{\infty}\left|\alpha_{n m}\right|^{2} e^{i \delta(n-m)}$ and $\langle\psi|N| \psi\rangle=$ $\sum_{n, m=0}^{\infty}\left|\alpha_{n m}\right|^{2}(n+m)$. We notice that both these expressions only depends on the squared modulus of the coefficients $\alpha_{n m}$ and so we can assume $\alpha_{n m}$ to be real and positive. The assumption that the devices are beamsplitters allows us to simplify the structure of the optimal input state for the tradeoff. First, we show that it is not restrictive to consider superpositions of the so called NOON [17] states.

Lemma 4. If $U$ is a beamsplitter, the optimal state $\left|\psi^{*}\right\rangle$ in Eq. (11) can be taken of the form

$$
\left|\psi^{*}\right\rangle=\sum_{n=0}^{\infty} \alpha_{n}\left|\phi_{n}\right\rangle, \quad\left|\phi_{n}\right\rangle=\sqrt{\frac{1}{2}}(|n, 0\rangle+|0, n\rangle)
$$

Proof. We show that for any state $|\psi\rangle=$ $\sum_{n, m=0}^{\infty} \alpha_{n m}|n, m\rangle$ there exists a state $\left|\psi^{\prime}\right\rangle=$ $\sum_{l=0}^{\infty} \alpha_{l}^{\prime}\left|\phi_{l}\right\rangle$ such that $\left\langle\psi^{\prime}|N| \psi^{\prime}\right\rangle \leq\langle\psi|N| \psi\rangle$ and $\left|\left\langle\psi^{\prime}|U| \psi^{\prime}\right\rangle\right| \leq|\langle\psi|U| \psi\rangle|$. Upon defining $\left|\alpha_{l}^{\prime}\right|^{2}=\sum_{|n-m|=l}\left|\alpha_{n m}\right|^{2}$, one can verify

$$
\begin{aligned}
\left\langle\psi^{\prime}|N| \psi^{\prime}\right\rangle & =\sum_{n, m=0}^{\infty} \alpha_{n m}^{2}|n-m| \leq\langle\psi|N| \psi\rangle, \\
\left|\left\langle\psi^{\prime}|U| \psi^{\prime}\right\rangle\right| & =\left|\sum_{n, m=0}^{\infty} \alpha_{n m}^{2} \cos (\delta|n-m|)\right| \leq \\
& \leq|\langle\psi|U| \psi\rangle|,
\end{aligned}
$$

that proves the statement.
From Eq. (14) it follows that the expectation value of $U$ over $|\psi\rangle$ is real. Following an argument similar to the one we used to prove Lemma 1 , the constraint $|\langle\psi|U| \psi\rangle|=K$ can be changed into $\langle\psi|U| \psi\rangle=K$ and Eq. (11) becomes

$$
\left|\psi^{*}\right\rangle=\arg \min _{|\psi\rangle \text { s.t. }\langle\psi|U| \psi\rangle=K}\langle\psi|N| \psi\rangle .
$$

One can observe that for any state $|\psi\rangle$ of the form of Eq. (14), the constraint $\sum_{n=0}^{\infty} \alpha_{n}^{2} \cos (\delta n)=K$ implies that there must exist at least one non-null coefficient $\alpha_{n}$ for $n$ s.t. $\cos (\delta n) \leq K$ and at least one for $n$ s.t. $\cos (\delta n) \geq K$. In the following lemma we will prove that not more than two non-null coefficient $\alpha_{n}$ are needed.

Lemma 5. The optimal state $\left|\psi^{*}\right\rangle$ that minimize Eq. (17) can be taken of the form

$$
\left|\psi^{*}\right\rangle=\alpha_{n_{1}}\left|\phi_{n_{1}}\right\rangle+\alpha_{n_{2}}\left|\phi_{n_{2}}\right\rangle
$$

Proof. Let us consider the optimal state $|\psi\rangle=$ $\sum_{n} \alpha_{n}\left|\phi_{n}\right\rangle$ with $\langle\psi|U| \psi\rangle=K$ and $\langle\psi|N| \psi\rangle=N_{\min }$. Suppose now that the set $\vec{\alpha}:=\left\{\alpha_{n}\right\}$ has $N \geq 3$ elements. Then there must exist $n_{1}$ and $n_{2}$ such that $\alpha_{n_{1}}, \alpha_{n_{2}} \neq 0$ and $\cos \left(\delta n_{1}\right) \leq K \leq \cos \left(\delta n_{2}\right)$. It is then possible to define $|\chi\rangle:=\beta_{n_{1}}\left|\phi_{n_{1}}\right\rangle+\beta_{n_{2}}\left|\phi_{n_{2}}\right\rangle$ such that $\langle\chi|U| \chi\rangle=K$. Furthermore, we can define $|\xi\rangle:=(1-\epsilon)^{-1 / 2} \sum_{n} \gamma_{n}\left|\phi_{n}\right\rangle$, where

$$
\gamma_{n}=\left\{\begin{array}{ll}
\alpha_{n} & \text { if } n \neq n_{1}, n_{2} \\
\sqrt{\alpha_{n}^{2}-\epsilon \beta_{n}^{2}} & \text { if } n=n_{1}, n_{2}
\end{array},\right.
$$

and $\epsilon \leq \min \left(\alpha_{n_{1}} / \beta_{n_{1}}, \alpha_{n_{2}} / \beta_{n_{2}}\right)$. We notice that $\langle\xi|U| \xi\rangle=K$, and

$$
N_{\min }=\epsilon\langle\chi|N| \chi\rangle+(1-\epsilon)\langle\xi|N| \xi\rangle .
$$

If $\langle\chi|N| \chi\rangle=N_{\min }$ the statement follows with $|\psi\rangle=$ $|\chi\rangle$. If $\langle\chi|N| \chi\rangle \neq N_{\min }$, either $\langle\chi|N| \chi\rangle<N_{\min }$ or $\langle\xi|N| \xi\rangle<N_{\min }$, that contradicts the hypothesis that $|\psi\rangle$ is the optimal state.

Since we both require $\langle\psi|U| \psi\rangle=K$ and $\alpha_{n_{1}}^{2}+\alpha_{n_{2}}^{2}=1$ the expression of the coefficients $\alpha_{n_{1}}, \alpha_{n_{2}}$ and of the mean value $\langle\psi|N| \psi\rangle$ are fixed by the choices of $n_{1}, n_{2}$ and $K$, i.e.

$$
\begin{aligned}
\alpha_{n_{1}} & =\sqrt{\frac{\cos \left(\delta n_{2}\right)-K}{\cos \left(\delta n_{2}\right)-\cos \left(\delta n_{1}\right)}}, \quad \alpha_{n_{2}}=\sqrt{1-\alpha_{n_{1}}^{2}} \\
\langle\psi|N| \psi\rangle & =\frac{n_{2} \cos \left(\delta n_{1}\right)-n_{1} \cos \left(\delta n_{2}\right)+K\left(n_{1}-n_{2}\right)}{\cos \left(\delta n_{1}\right)-\cos \left(\delta n_{2}\right)} .
\end{aligned}
$$

It is now convenient to rephrase the problem at hand in a geometrical way (see Fig. 11). Let us introduce the map $\mathrm{f}: \mathbb{N} \rightarrow \mathbb{R}^{2}$, defined as $\mathrm{f}(n)=(\cos (\delta n), n)$. We can associate to any couple $n_{1}$ and $n_{2}$ the line $L_{n_{1}, n_{2}}:=$ $\left\{t \mathrm{f}\left(n_{1}\right)+(1-t) \mathfrak{f}\left(n_{2}\right) \mid t \in \mathbb{R}\right\}$ and the segment $l_{n_{1}, n_{2}}:=$ 
$\left\{t \mathrm{f}\left(n_{1}\right)+(1-t) \mathrm{f}\left(n_{2}\right) \mid 0 \leq t \leq 1\right\}$. It is easy to prove that the expectation value $\langle\psi|N| \psi\rangle$ in Eq. (20) can be rewritten as

$$
\langle\psi|N| \psi\rangle=\left(\ell_{n_{1}, n_{2}} \cap r_{K}\right)_{y}
$$

where we defined the line $r_{K}:=\{(K, y) \mid y \in \mathbb{R}\}$ and the mapping $(a, b)_{y}=b$. For any $K$ it is then possible to define a partial ordering $<_{K}$ among the segments $l_{n_{1}, n_{2}}$ for which $\cos \left(\delta n_{1}\right) \leq K \leq \cos \left(\delta n_{2}\right)$, as follows:

$$
l_{n_{1}, n_{2}}>_{K} l_{n_{1}^{\prime}, n_{2}^{\prime}} \text { if }\left(l_{n_{1}, n_{2}} \cap r_{K}\right)_{y}>\left(l_{n_{1}^{\prime}, n_{2}^{\prime}} \cap r_{K}\right)_{y}
$$

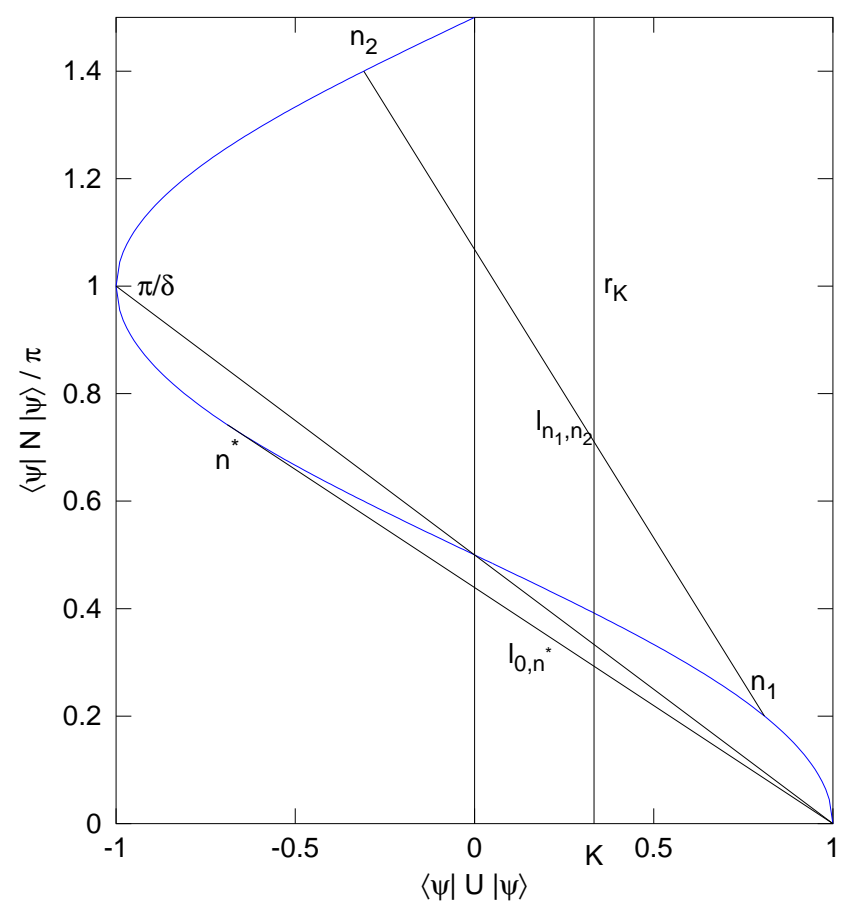

FIG. 1: (Color online) Geometrical representation of the optimization problem. For fixed values $n_{1}$ and $n_{2}$, each state of the form (18) is represented by a point of the line segment $l_{n_{1}, n_{2}}$ which extrema are $\left(\cos \left(\delta n_{1}\right), n_{1}\right)$ and $\left(\cos \left(\delta n_{2}\right), n_{2}\right)$. The $\mathrm{x}$ and $\mathrm{y}$ coordinates represent the expectation values $\langle\psi|U| \psi\rangle$ and $\langle\psi|N| \psi\rangle$, respectively. From the picture it is clear that the line segment $l_{0, n^{*}}$ lies below any other line segment $l_{n_{1}, n_{2}}$, and so it identifies the family of optimal states, as we prove in Prop. 1 .

We are now ready to proof the following Proposition. Here $\lfloor x\rfloor(\lceil x\rceil)$ denotes the minimum (maximum) integer number greater (smaller) than $x$.

Proposition 1. For any $K, 0 \leq K \leq 1$, the optimal state $\left|\psi^{*}\right\rangle$ that minimizes Eq. (17) is

$$
\left|\psi^{*}\right\rangle=\alpha_{n^{*}}\left|\phi_{n^{*}}\right\rangle+\alpha_{0}|00\rangle,
$$

where $n^{*}=\arg \min _{\lfloor\tilde{n}\rfloor,\lceil\tilde{n}\rceil}\left\langle\psi^{*}|N| \psi^{*}\right\rangle$, with $\tilde{n}$ the minimum positive solution of $\delta n=\tan (\delta n / 2)$.

Proof. First let us introduce the set $\Omega:=\{n \in \mathbb{N} \mid \pi / 2 \delta \leq$ $\left.n^{\prime} \leq \pi / \delta\right\}$. Since $\delta<\pi$ we observe that $\Omega$ must be nonempty. Consider now the lines $L_{n^{\prime}, 0}$ and $L_{n^{\prime}, m}$. It is easy to verify that for all $m$ we have $\left(L_{n^{\prime}, m} \cap r_{1}\right)_{y}>$ $\left(L_{n^{\prime}, 0} \cap r_{1}\right)_{y}$ which implies $\left(L_{n^{\prime}, m} \cap r_{K}\right)_{y}>\left(L_{n^{\prime}, 0} \cap r_{K}\right)_{y}$ for all $\cos \left(\delta n^{\prime}\right) \leq K \leq 1$ and finally $l_{n^{\prime}, m}>_{K} l_{n^{\prime}, 0}$ for all $\cos \left(\delta n^{\prime}\right) \leq K \leq \cos (\delta m)$. Similarly one can verify the bound $l_{n^{\prime}, 0}<_{K} l_{\pi / \delta, 0}<_{K} l_{n_{1}, n_{2}}$ that holds for $\cos \left(\delta n_{1}\right) \leq K \leq \cos \left(\delta n_{2}\right), n^{\prime} \in \Omega$. We can then restrict the optimization over the finite set of states $\left.S:=\{|\psi\rangle|| \psi\rangle=\alpha_{n^{\prime}}\left|\phi_{n^{\prime}}\right\rangle+\alpha_{0}|00\rangle, n^{\prime} \in \Omega\right\}$. As a consequence, the mean value $\langle\psi|N| \psi\rangle$ becomes

$$
\langle\psi|N| \psi\rangle=\frac{(1-K) n}{1-\cos (\delta n)} .
$$

The right hand side of Eq. (25) can be proven to be a convex function [18] for $\pi / 2 \leq \delta n \leq \pi$ and achieves its minimum for $\tilde{n}$ minimum positive solution of $\delta n=$ $\tan (\delta n / 2)$. Since $\tilde{n}$ is in general not integer the optimal value $n^{*}$ is given by $\arg \min _{\lfloor\tilde{n}\rfloor,\lceil\tilde{n}\rceil}\left\langle\psi^{*}|N| \psi^{*}\right\rangle$.

Figure 2 shows the optimal energy-error tradeoff obtained with the discrimination strategy of Prop. 1.

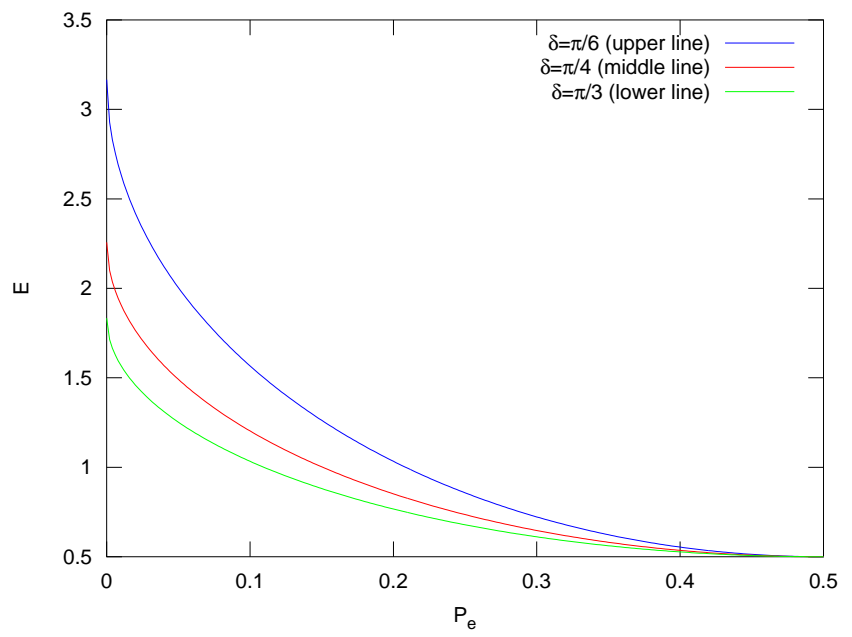

FIG. 2: (Color online) Optimal tradeoff between the energy $E$ and the probability of error $P_{e}$ in the discrimination of I and $U=\exp \left(i\left(\delta a_{1}^{\dagger} a_{1}-\delta a_{2}^{\dagger} a_{2}\right)\right)$, for various values of $\delta$.

\section{B. An iterative algorithm for the general case}

Here we provide an iterative steepest-descent algorithm [19] to find a state $|\psi\rangle$ that solves the optimization problem in Eq. (111). With the same argument we use in the proof of Lemma 2 we can rephrase the optimization problem in Eq. (11) as the minimization of the convex combination

$$
C(\psi):=p\langle\psi|N| \psi\rangle+(1-p)|\langle\psi|U| \psi\rangle|^{2} .
$$

We are now ready to introduce the iterative procedure. 
Proposition 2. The following algorithm converges to a state $|\psi\rangle$ that is optimal according to Eq. (26). Take an arbitrary state $\left|\psi^{0}\right\rangle$. Given $\left|\psi^{n}\right\rangle$, evaluate $\left|\psi^{n+1}\right\rangle$ by the following steps:

1. Evaluate the derivative of the figure of merit $C\left(\psi^{n}\right)$

$$
\begin{gathered}
\frac{\partial C\left(\psi^{n}\right)}{\partial\left\langle\psi^{n}\right|}=\left[p N+(1-p)\left(\left\langle\psi^{n}|U| \psi^{n}\right\rangle U^{\dagger}+\right.\right. \\
\left.\left.+\left\langle\psi^{n}\left|U^{\dagger}\right| \psi^{n}\right\rangle U\right)\right]\left|\psi^{n}\right\rangle .
\end{gathered}
$$

2. Pick up a small enough positive $\alpha$ and evaluate

$$
\left|\hat{\psi}^{n+1}\right\rangle=(1-\alpha)\left|\psi^{n}\right\rangle-\alpha \frac{\partial C\left(\psi^{n}\right)}{\partial\left\langle\psi^{n}\right|} .
$$

3. Normalize $\left|\hat{\psi}^{n+1}\right\rangle$ according to

$$
\left|\psi^{n+1}\right\rangle=\left|\hat{\psi}^{n+1}\right|^{-1}\left|\hat{\psi}^{n+1}\right\rangle \text {. }
$$

Proof. The algorithm in Prop. 2 is a steepest-descent algorithm: we move the state in the direction of the gradient of the figure of merit $C(\psi)$, so by construction one has $C\left(\psi^{n+1}\right) \leq C\left(\psi^{n}\right)$.

The parameter $\alpha$ controls the length of each iterative step, so for $\alpha$ too large an overshooting can occur. This can be kept under control by evaluating the figure of merit $C(\psi)$ at the end of each step: if $C(\psi)$ increases instead of decreasing, we are warned that we have taken $\alpha$ too large. Figure 3 shows the optimal energy-error tradeoff obtained with the algorithm of Prop. 2

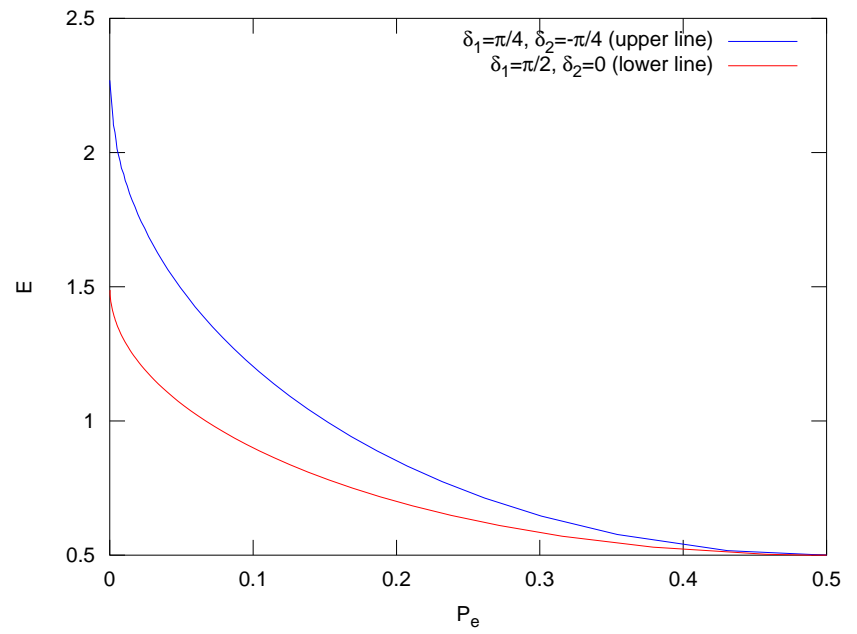

FIG. 3: (Color online) Optimal tradeoff between the energy $E$ and the probability of error $P_{e}$ in the discrimination of two linear passive optical devices $I$ and $U$. We consider the case in which $U$ is a two mode device, namely $U=\exp \left(i\left(\delta_{1} a_{1}^{\dagger} a_{1}+\right.\right.$ $\left.\left.\delta_{2} a_{2}^{\dagger} a_{2}\right)\right)$.

\section{Discrimination of passive devices with limited resources}

Here we consider the minimum energy discrimination that makes use of coherent input states $\left|\alpha_{i}\right\rangle$ and homodyne detections $X_{\varphi_{i}}$ to discriminate a single use of a $n$ modes passive linear optical device randomly chosen in the set $\{I, U\}$ with equal probabilities.

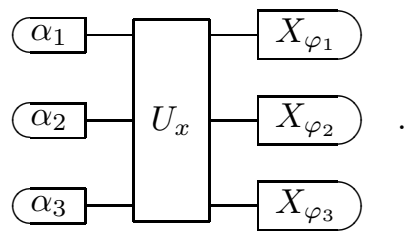

If we consider coherent input states $\left|\alpha_{i}\right\rangle$ on mode $i$ the global input state is $|\xi\rangle=\bigotimes_{i}\left|\alpha_{i}\right\rangle$ which corresponds to an energy value

$$
E(\xi):=\langle\xi|E| \xi\rangle=\frac{1}{2}+\langle\xi|N| \xi\rangle=\frac{1}{2}+\sum_{i}\left|\alpha_{i}\right|^{2}
$$

Since for any passive linear device $V$ we have that $V \bigotimes_{i}\left|\alpha_{i}\right\rangle=\bigotimes_{i}\left|\beta_{i}\right\rangle$ with $\left|\beta_{i}\right\rangle$ are coherent state, we can assume $U$ to be diagonal, i.e. $U=\sum_{i} e^{i \delta_{i} a_{i}^{\dagger} a_{i}}$. The evolution of $|\xi\rangle$ under the action of $U$ is then given by

$$
U|\xi\rangle=\bigotimes_{i}\left|e^{i \delta_{i}} \alpha_{i}\right\rangle
$$

A quantum homodyne detection $X_{\varphi}$ is described 14 , 20, 21] by the POVM $\{|x, \varphi\rangle\langle x, \varphi|\}$ where $|x, \varphi\rangle$ are the eigenstate of the quadrature $e^{i \varphi} a+e^{-i \varphi} a^{\dagger}$. The probability of outcome $x$ when the system is prepared in a coherent state $|\alpha\rangle, \alpha=e^{i \phi_{\alpha}}|\alpha|$ is given by the Gaussian

$$
p_{\varphi}(x \mid \alpha)=|\langle\alpha \mid x, \varphi\rangle|^{2}=\frac{1}{\sqrt{2 \pi}} e^{-\frac{1}{2}\left(x-2|\alpha| \cos \left(\varphi+\phi_{\alpha}\right)\right)^{2}} .
$$

We notice that $p_{\varphi}(x \mid \alpha)$ depends on $\varphi$ only trough the $\operatorname{sum} \varphi+\phi_{\alpha}$. We can then fix $\varphi=0$ and vary only the $\alpha_{i}$. conditional probabilities of outcome $\mathbf{x}=\left(x_{i}\right)$ given $I$ or $U$ are $n$-dimensional Gaussians, namely

$$
\begin{aligned}
& p(\mathbf{x} \mid I)=(2 \pi)^{-n / 2} e^{\left|\mathbf{x}-\mathbf{v}_{\mathbf{0}}\right|^{2} / 2}, \\
& p(\mathbf{x} \mid U)=(2 \pi)^{-n / 2} e^{\left|\mathbf{x}-\mathbf{v}_{\mathbf{1}}\right|^{2} / 2}, \\
& \mathbf{v}_{\mathbf{0}}=\left(2 \operatorname{Re} \alpha_{i}\right), \quad \mathbf{v}_{\mathbf{1}}=\left(2 \operatorname{Re} e^{i \delta_{i}} \alpha_{i}\right),
\end{aligned}
$$

Any classical postprocessing of the outcome $\mathbf{x}$ can be described by a function $q(X \mid \mathbf{x})$ that evaluates to 1 if one guess the unitary $X$ from outcome $\mathbf{x}$, and to 0 otherwise, with $X=I, U$. The probability of error is given by

$$
P_{e}(\xi)=\frac{1}{2} \int d \mathbf{x} p(\mathbf{x} \mid I) q(U \mid \mathbf{x})+p(\mathbf{x} \mid U) q(I \mid \mathbf{x}),
$$


and thus the optimal postprocessing is

$$
q(X \mid \mathbf{x})=\left\{\begin{array}{l}
1 \text { if } p(\mathbf{x} \mid X) \geq p(\mathbf{x} \mid Y) \\
0 \text { if } p(\mathbf{x} \mid X)<p(\mathbf{x} \mid X)
\end{array} .\right.
$$

Inserting Eq. (37) and Eq. (35) into the expression (36), the probability of error becomes

$$
P_{e}(\xi)=\frac{1}{2}+\frac{(2 \pi)^{-n / 2}}{2} \int_{A} d \mathbf{x} e^{-\frac{\left|\mathbf{x}-\mathbf{v}_{\mathbf{0}}\right|^{2}}{2}}-e^{-\frac{\left|\mathbf{x}-\mathbf{v}_{1}\right|^{2}}{2}},
$$

where we defined the set

$$
A=\left\{\mathbf{x} \text { s.t. }\left|\mathbf{x}-\mathbf{v}_{\mathbf{0}}\right|^{2} \geq\left|\mathbf{x}-\mathbf{v}_{\mathbf{1}}\right|^{2}\right\} .
$$

Within this framework it is more convenient to fix the amount of energy, that is the average number of photons $\eta$, and find the input state $\left|\xi^{*}\right\rangle$ that minimizes the probability of error in the discrimination, i.e.

$$
\left|\xi^{*}\right\rangle=\arg \min _{\langle\xi|N| \xi\rangle=\eta} P_{e}(\xi)
$$

With a little machinery it is possible to prove that $P_{e}(|\xi\rangle)$ is a non-increasing function of $\left|\mathbf{v}_{\mathbf{0}}-\mathbf{v}_{\mathbf{1}}\right|^{2}$ and then the minimization of $P_{e}(|\xi\rangle)$ can be rephrased as a maximization of $\left|\mathbf{v}_{\mathbf{0}}-\mathbf{v}_{\mathbf{1}}\right|^{2}$. We have then

$$
\begin{aligned}
\left|\mathbf{v}_{\mathbf{0}}-\mathbf{v}_{\mathbf{1}}\right|^{2} & =4 \sum_{i}\left[\operatorname{Re}\left(\alpha_{i}\right)-\operatorname{Re}\left(e^{i \delta} \alpha_{i}\right)\right]^{2}= \\
& =4 \sum_{i}\left[\left(\cos \left(\phi_{i}\right)-\cos \left(\phi_{i}+\delta_{i}\right)\right)\left|\alpha_{i}\right|\right]^{2} \leq \\
& \leq 4 \sum_{i}\left[2 \sin \left(\delta_{i} / 2\right)\left|\alpha_{i}\right|\right]^{2} \leq 16 \sin ^{2}(\delta / 2) \eta
\end{aligned}
$$

where $\delta^{*}:=\arg \max _{\delta_{i}}\left|\delta_{i}\right|$, and $i^{*}$ labels the corresponding mode. The bounds in Eq. (41) are achieved for

$$
\left|\xi^{*}\right\rangle=\bigotimes_{i \neq i^{*}}\left|0_{i}\right\rangle \otimes\left|\alpha_{i^{*}}^{*}\right\rangle
$$

where $\alpha_{i^{*}}^{*}=\sqrt{\eta} \exp \left(i \frac{\pi-\delta^{*}}{2}\right)$. The corresponding optimal discrimination strategy is

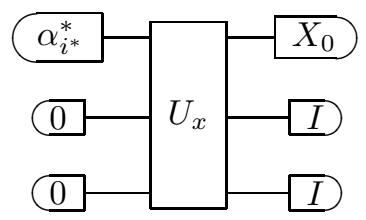

where $-I$ means that the corresponding mode is discarded. With this choice of the input state the probability of error becomes

$$
P_{e}=\frac{1}{2}\left(1+\Phi\left(-2 \sqrt{\eta} \sin \frac{\left|\delta^{*}\right|}{2}\right)-\Phi\left(2 \sqrt{\eta} \sin \frac{\left|\delta^{*}\right|}{2}\right)\right)
$$

where $\Phi(x)=\frac{1}{\sqrt{2 \pi}} \int_{-\infty}^{x} d t \exp \left(-t^{2} / 2\right)$ denotes the normal cumulative distribution function.
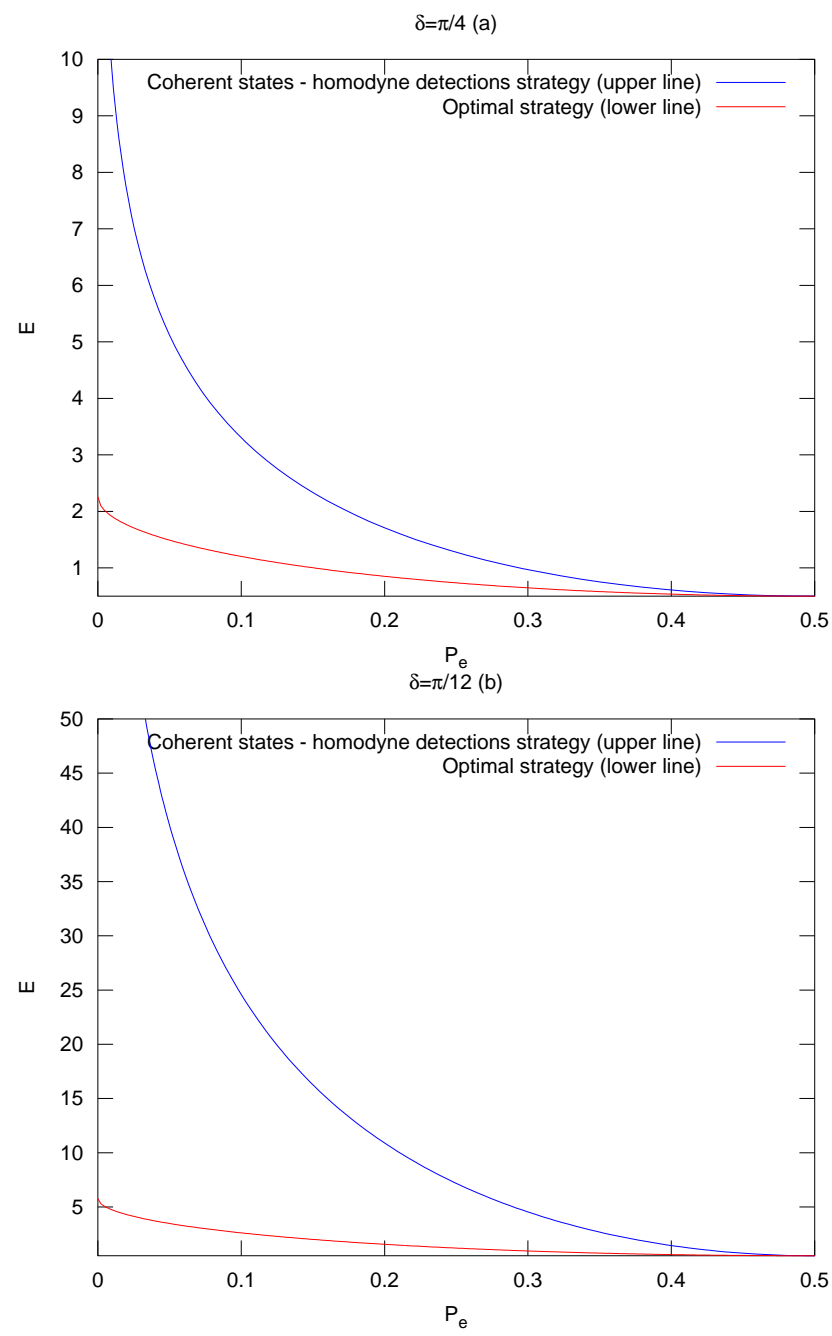

FIG. 4: (Color online) Optimal tradeoff between the energy $E$ and the probability of error $P_{e}$ in the discrimination of $I$ and $U=\exp \left(i\left(\delta a_{1}^{\dagger} a_{1}-\delta a_{2}^{\dagger} a_{2}\right)\right)(\delta=\pi / 4$ in (a) and $\delta=\pi / 12$ in (b)). The upper line represents the discrimination with coherent states and homodyne detections, while the lower line represents the optimal discrimination. Comparing (a) and (b), we notice that the improvement provided by the optimal strategy increases as $\delta$ decreases.

From Eq. (44) one can obtain the tradeoff between the energy and the probability of error, which is plotted in Fig. 4, for some choices of $U_{1}$ and $U_{2}$. If we consider the case in which we want to discriminate a 50/50 beamsplitter from the identity, one can notice that, for $P_{e}=0.1$, the coherent state - homodyne detection discrimination strategy requires a factor of $\sim 4$ more photons that the optimal strategy. Moreover, this factor increases as the two devices get closer, i. e. for small values of $\delta$. For example, when $\delta=\pi / 12$, the factor is $\sim 12$. As expected, one notice that this factor increases when the probability of error decreases. 


\section{CONCLUSION}

In this paper we studied the energy-error tradeoff in the discrimination of two linear passive optical devices. We derived the optimal strategy when no restrictions in the input state and in the final measurement are assumed. It is shown that the input state can be taken as pure and no ancillary modes are needed. For the beamsplitter case the input state is proved to be a coherent superposition of a NOON state $\left|\phi_{n^{*}}\right\rangle$ and the vacuum $|00\rangle$. It is worth noting that the choice of $\left|\phi_{n^{*}}\right\rangle$ depends only upon the reflectivity of the beamsplitter. We provided an iterative algorithm to solve the problem in the general case.

In Section $\Pi$ we considered the practical scenario in which one performs the discrimination with coherent in- put states and homodyne detections. This strategy, when compared with the optimal one, turns out to be largely suboptimal. For example, for a 50/50 beamsplitter the energy required for the discrimination when the probability of error is 0.1 is 4 times smaller with the optimal strategy than with the coherent-homodyne one.

\section{Acknowledgments}

We thank Paolo Perinotti, Massimiliano F. Sacchi, and Michal Sedlák for useful suggestions and discussions. This work is supported by Italian Ministry of Education through PRIN 2008 and the European Community through the COQUIT and CORNER projects.
[1] S. Lloyd, Science 321, 1463 (2008)

[2] S. H. Tan, B. I. Erkmen, V. Giovannetti, S. Guha, S. Lloyd, L. Maccone, S. Pirandola, J. H. Shapiro, Phys. Rev. Lett. 101, 253601 (2008).

[3] J. H. Shapiro and S. Lloyd, New J. Phys. 11063045 (2009).

[4] A. Acin, Phys. Rev. Lett 87, 177901 (2001).

[5] G. M. D' Ariano, P. Lo Presti, and M. G. A. Paris, J. Opt. B 4, 273 (2002).

[6] R. Duan, Y. Feng, M. Ying, Phys. Rew. Lett. 98, 100503 (2007).

[7] M. Ziman and M. Sedlák, Journal of Modern Optics, 57, 253 (2010).

[8] D. Collins, Phys. Rev. A 81, 052323 (2010).

[9] The scenario in which multiple uses are considered involves the optimization over all the possible strategies (parallel, sequential, hybrid), and will be discussed in a future work.

[10] C. W. Helstrom, Quantum Detection and Estimation Theory (Academic Press, New York, 1976).
[11] S. Pirandola, Phys. Rev. Lett. 106, 090504 (2011).

[12] R. Nair, arXiv:quant-ph/1105.4063v2 (2011).

[13] C. Invernizzi, M. G. A. Paris, and S. Pirandola, arXiv:quant-ph/1011.2785v4 (2010).

[14] W. Vogel and D. G. Welsch, Quantum Optics (WileyVCH, Weinheim, 2006).

[15] U. Leonhardt, Rept. Prog. Phys. 66, 1207 (2003).

[16] I. L. Chuang, M. A. Nielsen, Quantum Information and Communication (Cambridge, Cambridge University Press, 2000).

[17] B. C. Sanders, Phys. Rev. A 40, 2417 (1989).

[18] S. Boyd and L. Vandenberghe, Convex Optimization (Cambridge, New York, 2004).

[19] G. Arfken, Mathematical Methods for Physicists (Academic Press, Orlando, 1985).

[20] T. Tyc, B. C. Sanders, J. Phys. A 37, 7341 (2004).

[21] K. Banaszek, K. Wodkiewicz, Phys. Rev. A 55, 3117 (1997). 